\title{
Frenotomia lingual em lactentes
}

\author{
Lingual frenotomy in infants
}

Iryana Marques Sena Procopio*

Vanessa Polina Pereira Costa**

Erica Negrini Lia**

\section{Resumo}

Objetivo: o objetivo deste trabalho foi realizar uma revisão de literatura acerca do freio lingual anômalo em lactentes, relacionando diagnóstico e tratamento. Revisão de literatura: esta anomalia limita os movimentos da língua durante o choro, lalação e pode gerar desconforto durante a amamentação. A metodologia do trabalho constituiu em buscas de artigos publicados em língua portuguesa e inglesa nas bases de dados Bireme, Google Acadêmico e PubMed, entre os anos de 2004 e 2016. Foram identificados 645 artigos através da combinação de palavras-chave, sendo que 29 foram utilizados por apresentarem resultados condizentes com o objetivo desta revisão. Considerações Finais: através dos estudos consultados não foi possível identificar um instrumento padrão-ouro que diagnostique a anquiloglossia em bebês, a despeito do fácil diagnóstico. Há controvérsias na literatura em relação ao tratamento por meio da frenotomia e sua contribuição para a meIhora na amamentação, movimentos da língua e desconforto da mãe ao amamentar, embora grande parte dos estudos demonstre benefícios evidentes.

Palavras-chave: Freio lingual. Anquiloglossia. Bebês.

\section{Introdução}

Anquiloglossia é uma anomalia de desenvolvimento da língua, que pode ocorrer de forma parcial ou total, na qual o freio lingual se encontra mais curto, resultando na limitação dos seus movimen$\operatorname{tos}^{1-3}$.

Em lactentes, a anquiloglossia pode gerar dificuldades na amamentação, uma vez que a deglutição e sucção estão relacionadas a este processo. Essa situação pode chegar a causar dificuldade de ganho de peso da criança e dor no mamilo da mãe durante o ato de amamentar, e assim, levar a um desmame precoce e prejuízo do desenvolvimento normal da criança ${ }^{2,4,5}$. Além disso, podem também ocorrer limitações do movimento da língua durante o choro e a lalação e posteriormente, dificuldades de dicção ${ }^{3}$. Em casos mais graves, pode levar também a deficiência de crescimento da mandíbula ${ }^{6}$.

O diagnóstico deve ser realizado precocemente, com o objetivo da intervenção cirúrgica para remoção do freio, a fim de otimizar a amamentação, garantindo assim conforto à criança e à mãe ${ }^{4}$. A resolução da anquiloglossia é cirúrgica, por meio

Pós-doutora em Odontopediatria- Professora Adjunta do Departamento de Odontologia- Faculdade de Ciências da Saúde- Universidade de Brasília- UnBBrasília, Brasil.

*** Pós-doutora em Ciências da Saúde- Professora Associada do Departamento de Odontologia- Faculdade de Ciências da Saúde- Universidade de Brasília- UnBBrasília, Brasil. 
da frenotomia, indicada quando o freio lingual restringe ou dificulta a movimentação da língua. Em lactentes, indica-se a frenotomia quando há dificuldades durante a amamentação ou o choro e lalação. Já em crianças maiores, a indicação se dá frente a problemas periodontais e/ou dificuldades de dicção ${ }^{3}$.

Os protocolos existentes para o diagnóstico de anquiloglossia ${ }^{7-10}$ não apresentam parâmetros claros e objetivos que indiquem normalidade ou anormalidade do freio lingual, e em quais situações a frenotomia estaria indicada, sendo portanto essa cirurgia baseada em critérios subjetivos ${ }^{11}$.

Como a cavidade bucal deve ser examinada ao nascimento do bebê, todos os profissionais, como médicos, enfermeiros, fonoaudiólogos, otorrinolaringologistas e cirurgiões-dentistas devem estar atentos à ocorrência da anquiloglossia, seu correto diagnóstico e intervenção quando necessária.

Portanto, a indicação da frenotomia em lactentes ainda é um assunto que deve ser explorado, visto as divergências de opiniões dos profissionais da área da saúde a despeito dos benefícios evidentes e baixo risco relacionado ao procedimento. Este trabalho teve como objetivo realizar uma revisão de literatura sobre a o freio lingual em lactentes, discorrendo sobre a importância do diagnóstico precoce da anquiloglossia e das vantagens da realização do procedimento em fases inicias da vida.

\section{Metodologia}

Foram realizadas buscas por artigos publicados em português e inglês entre julho de 2004 e novembro de 2016, nas bases de dados PubMed, Google Acadêmico e Bireme, usando as palavras-chave: frenectomia lingual, anquiloglossia, bebês, breastfeeding, lingual frenulum, ankyloglossia, baby and tongue-tie.

Com a combinação das palavras-chave foram encontrados: 23 artigos no Pubmed, 601 no Google Acadêmico e 21 na Bireme. Foram selecionados 29 artigos porque estes apresentavam resultados condizentes com o objetivo desta revisão, sendo 2 relatos de caso, 1 série de casos, 1 revisão metodológica, 9 estudos transversais, 3 estudos clínicos, 2 estudos de caso-controle, 1 estudo de coorte, 1 parecer técnico- científico, 4 ensaios clínicos randomizados, 1 ensaio clínico prospectivo, 4 revisões sistemáticas. Além dos artigos foram utilizados 2 livros para a realização desta revisão.

\section{Revisão de Literatura}

\section{Freio lingual: desenvolvimento e anatomia}

A língua inicia seu desenvolvimento na quarta semana de vida intrauterina, quando os arcos faríngeos se encontram na linha média, onde surgem as protuberâncias linguais laterais. Elas se alargam rapidamente e se encontram umas com as outras e com o tubérculo ímpar para formar uma grande massa da qual é formada a mucosa dos dois terços anteriores da língua ${ }^{12}$.

Depois de formada a base da língua, ocorre a morte celular programada e reabsorção do músculo esquelético em desenvolvimento na sua região anterior ventral e, normalmente, uma fina faixa de tecido permanece como o único elo de ligação, formando o freio lingual. Distúrbios deste processo podem resultar na inserção anteriorizada do freio lingual, mais larga e/ou encurtada, gerando a anquiloglos$\operatorname{sia}^{13}$.

O freio lingual conecta a língua ao assoalho da boca. É uma prega mediana de túnica mucosa que passa da gengiva, recobrindo a face lingual da crista alveolar anterior, para a face póstero-anterior da língua. É constituído de tecido conjuntivo fibrodenso e, ocasionalmente, de fibras superiores do músculo genioglosso. Esta prega é responsável pela mobilidade da língua e se estende de uma parte mais fixa para uma parte com maior liberdade de movimentos $^{3,4}$.

\section{Sucção e deglutição do recém-nascido e lactente}

O reflexo de sucção manifesta-se logo ao nascimento e perdura até os 2 meses de idade, mas a amamentação é um processo que se prolonga, sendo que a Organização Mundial da Saúde recomenda que se estenda até os 2 anos de idade ${ }^{14}$.

A extração do leite do seio materno exige do bebê esforço e posicionamento correto. Na postura correta durante a amamentação, o bebê faz a abertura ampla da boca, abocanhando o mamilo a aproximadamente 2 a $3 \mathrm{~cm}$ da aréola mamária, realizando vedamento perfeito entre as estruturas orais do lactente e a mama da sua mãe, o que é importante para a formação do vácuo intra-oral. $\mathrm{O}$ ápice da língua permanece na região anterior para garantir o vedamento da boca ao mamilo do lactante. Portanto, dentre outras estruturas, a língua assume um papel de grande importância na sucção durante a amamentação ${ }^{15}$.

Suas bordas se elevam lateralmente, juntamente com o ápice, em formato de concha, facilitando a captação do leite. O leite é então depositado sobre a região posterior da língua, e é gerado um movimento peristáltico rítmico em direção à orofaringe, culminando com a deglutição. Desta maneira, o leite consegue ser extraído sem uso de mecanismos de força, que são capazes de causar desconfortos na amamentação $^{15}$. 


\section{Anquiloglossia - Definição e características clínicas}

A anquiloglossia é uma anomalia de desenvolvimento da língua caracterizada pela inserção curta do freio lingual, ou inserção extensa do músculo genioglosso, o que resulta na limitação dos movimentos linguais ${ }^{1}$, como ilustrado na Figura 1 . A sua etiopatogenia é desconhecida, mas existem estudos que associam a anquiloglossia a mutações genéticas, acompanhadas ou não por outras alterações congênitas, como a fenda palatina e hipodontia ${ }^{16}$.

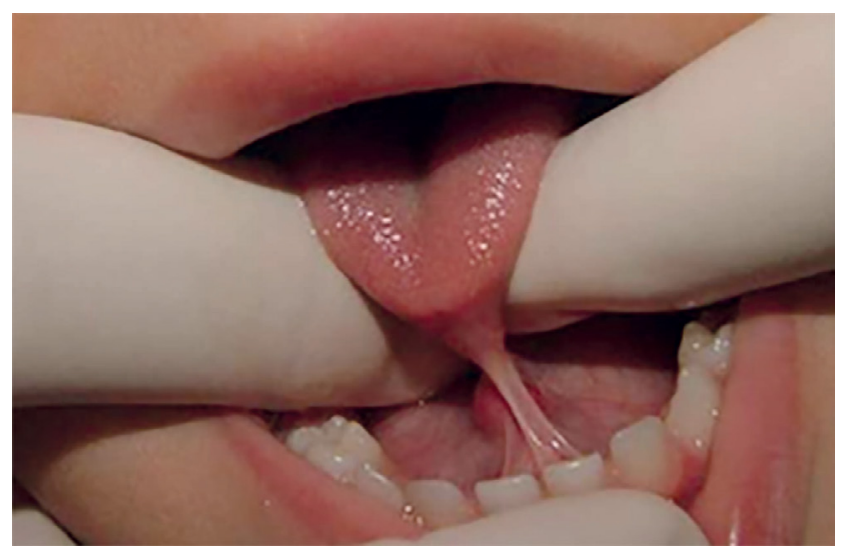

Figura 1 - Freio lingual de inserção curta, caracterizando anquiloglossia

As características clínicas preponderantes são: freio lingual anormalmente curto, inserido próximo ou no ápice lingual, dificuldade de elevação da língua e de tocar o palato, dificuldade de protrusão lingual, acima de 1 a $2 \mathrm{~mm}$ além dos incisivos centrais inferiores (durante a protrusão a língua assume "formato de coração") dificuldade de realização de movimentos de lateralidade linguais ${ }^{1}$.

Esta anomalia pode resultar em limitações dos movimentos da língua, que pode interferir na fala, amamentação e na higienização oral do bebề ${ }^{2,17}$. Por causar a diminuição dos movimentos da língua, a anquilglossia afeta a amamentação do lactente, o vedamento do mamilo com a língua é inadequado, e pode gerar dor e desconforto à mãe na hora da amamentação. Com a amamentação prejudicada, o lactente pode apresentar dificuldade em ganhar peso $0^{2,3,17}$.

\section{Prevalência e classificação}

Dependendo do critério de diagnóstico utilizado, e da população de estudo, a prevalência da anquiloglossia na literatura varia de menos de $1 \%{ }^{18}$ a $10,7 \%^{19}$. Não há uniformidade de definição diagnóstica e os dados da literatura são provenientes em grande parte de estudos observacionais ou de série de $\operatorname{casos}^{16}$. Quanto ao gênero, na maioria dos estudos, o sexo masculino parece ter maior prevalência de anquiloglossia comparado ao sexo feminino ${ }^{19-21}$.

Hazelbaker et al. ${ }^{7}$ (1993) propuseram uma ferramenta para avaliação da severidade da anquilo- gossia em lactentes denominada Hazelbaker Assessment Tool for Lingual Frenulum Function (HATLFF). Esta ferramenta calcula escores de acordo com 5 itens que avaliam a aparência da língua do lactente e 7 itens que avaliam sua função. Após avaliar e somar os escores, os valores encontrados são correlacionados a 3 categorias: "Perfeito", "Aceitável" ou de "Função Prejudicada".

O estudo de Kotlow ${ }^{8}$ (1999) descreve medidas objetivas aceitáveis para o frênulo lingual de bebês e jovens até 14 anos, com classificação de severidade, apesar de não apresentar um protocolo completo de avaliação do frênulo lingual.

Martinelli et al. ${ }^{9}$ (2013) apresentaram um novo protocolo de avaliação do frênulo lingual para bebês, relacionando aspectos anatômicos e funcionais. O protocolo é composto por três partes, tais como a História Clínica (aspectos hereditários e perguntas à mãe sobre a amamentação); Avaliação Anatomo-funcional, Avaliação da Sucção Não-Nutritiva (inspeção da sucção com dedo do avaliador enluvado) e Suç̧ão Nutritiva (com observação da amamentação durante 5 minutos).

O Bristol Tongue Assessment Tool (BTAT) foi um instrumento recentemente validado por Ingram et al. ${ }^{10}$ (2015) com escores e classificação de severidade do funcionamento da língua, desenvolvido com base em prática clínica e comparação ao HATLFF. Este instrumento fornece uma objetiva, clara e simples medida da severidade de anquiloglossia, para selecionar os lactentes para frenotomia e monitorar o efeito do procedimento.

\section{Tratamento cirúrgico da anquiloglossia}

O tratamento cirúrgico da anquiloglossia deve ser realizado através da frenectomia que consiste na remoção do tecido mucoso que compõem o freio lingual. Entretanto, em recém-nascidos e lactentes pode ser realizada a frenotomia, que consiste na incisão linear anteroposterior do freio lingual, sem remoção de tecido ${ }^{22}$.

Ambos são procedimentos simples com baixa possibilidade de complicações pós-operatórias. A técnica empregada na frenotomia consiste de anestesia tópica da mucosa do freio lingual, seguida de incisão de 3 a 4 milímetros de profundidade na região mais delgada do mesmo, por ser pouco vascularizada $^{5}$. Os benefícios do procedimento para o bebê são melhora na postura e mobilidade da língua, e na postura do lábio o que contribui para garantir os benefícios da amamentação, como o ganho de peso, através do correto vedamento do lábio no mamilo ${ }^{23}$. Além disso, o ato de amamentar se torna mais prazeroso para a mãe, diante da ausência da dor.

Um estudo com 24 mães e lactentes de aproximadamente 28 dias foi realizado para mensuração do impacto da frenotomia lingual sobre a produção de leite durante 24 horas. Foram realizadas ultras- 
sonografias submentuais da cavidade oral dos bebês, antes e após 7 dias da realização da frenotomia para avaliação da transferência do leite, presença de dor, deglutição audível, e aspecto do mamilo. Observou-se que houve melhora significativa do padrão de amamentação após a frenotomia, pois os bebês passaram a comprimir menos o mamilo com a língua e, com isso, houve melhora do vedamento entre a boca da criança e o seio materno, otimizando a transferência do leite e causando menos dor à mãe no processo ${ }^{5}$.

Um ensaio clínico randomizado realizado por Berry et al. ${ }^{24}$ (2012) avaliou bebês nascidos em uma maternidade do Reino Unido com idade entre 5 a 115 dias (média 32 dias; mediana, 23 dias). Os bebês foram divididos em dois grupos, sendo o grupo de estudo submetido a frenotomia sem o conhecimento das mães sobre a realização do procedimento e o segundo grupo não submetido à cirurgia (grupo controle). Cerca de $78 \%$ das mães dos bebês alocados no grupo que recebeu a cirurgia relataram melhora da amamentação imediatamente após a frenotomia; contra $47 \%$ das mães do grupo controle. No dia seguinte, $90 \%$ das mães dos bebês do grupo de estudo relataram melhora da amamentação, porcentagem que perdurou até o término do estudo, 3 meses após o procedimento. Entretanto, após os 3 meses, apenas $51 \%$ das mães dos bebês do grupo controle afirmaram melhora da amamentação. De acordo com o estudo, a melhora dos parâmetros de amamentação parece não ser relacionada a efeito placebo.

Em contrapartida, outro estudo também realizado no Reino Unido ${ }^{25}$, avaliou a eficácia da frenotomia em bebês com idade inferior a 2 semanas com grau leve a moderado de anquiloglossia, cujas mães relataram dificuldades de amamentação. Os resultados demonstraram que a frenotomia precoce não resultou em melhoras objetivas dos parâmetros da amamentação. Os autores também relataram dificuldades de mensuração do grau de anquiloglossia e mensuração do impacto da frenotomia.

Um estudo de coorte desenvolvido por Haham et al. ${ }^{26}$ (2014) não foi suficientemente consistente para afirmar qual tipo de freio lingual, classificado de acordo com Coryllos e Salloum ${ }^{27}$ (2016), apresenta relação com problemas de amamentação. No entanto, os resultados foram coletados com pouco tempo de acompanhamento ( 2 semanas) e o tipo de classificação usada, também pode ser questionada.

Uma revisão sistemática realizada recentemente sobre o efeito do tratamento da anquiloglossia sobre a amamentação revelou que é pequena a evidência de que a frenectomia possa estar associada com o relato de melhora na amamentação pelas mães, uma vez que os estudos apresentam metodologia inconsistente e com pequeno tempo de acompanhamento ${ }^{28}$.

No entanto, o tipo de cirurgia realizada, se simples liberação do freio (frenulotomia) ou Z-plasty (frenuloplastia) parece exercer o mesmo efeito positivo sobre amamentação ou sucção, apesar de a Z-plasty ter demonstrado um efeito maior na articulação, no movimento da língua e na satisfação da mãe do que a simples liberação, além de menor dor nas mamas ${ }^{29}$.

Muitas vezes, os odontólogos e otorrinolaringologistas tendem a realizar a cirurgia para corrigir as alterações do freio lingual. Já o fonoaudiólogo, avalia as condições do freio, verificando os movimentos da língua e avaliando as funções de mastigação, deglutição e fala, optando por terapia conservadora na grande maioria dos casos, ou complementar à cirurgia ${ }^{30}$.

Entretanto, não é frequente a indicação da cirurgia nos primeiros anos de vida da criança, o que pode levar a consequências futuras para o paciente. Há estudos que relacionam a respiração bucal, mordida aberta anterior e anquiloglossia. Dentre outras consequências estão as alterações oclusais e periodontais e dificuldade de movimentação da língua. Pode-se observar também, alterações de deglutição, mastigação e dicção ${ }^{3}$.

\section{Complicações pós-cirúrgicas}

O procedimento é rápido com pouco ou nenhum sangramento, desde que a incisão seja realizada corretamente. As complicações atribuídas à frenotomia podem ser infecção e hemorragia causada pelo rompimento da artéria lingual. A hemostasia é realizada por meio da compressão por gaze e através da colocação da criança imediatamente para a amamentação ${ }^{2}$.

Berry et al. ${ }^{24}$ (2012) demonstraram que não houve complicações importantes após a realização da frenotomia em 60 bebês de 5 a 115 dias de idade. Apenas $5 \%$ das mães reportaram um pequeno sangramento relacionado ao procedimento, até chegarem em casa.

Griffiths et al. ${ }^{31}$ (2004) reportaram a presença de úlcera, depois de $48 \mathrm{~h}$ no local da divisão do freio em 4 pacientes de um total de 215 pacientes (1\%), com menos de 3 meses de idade e dor duradoura por mais de 24 horas em 1 paciente $(0,5 \%)$.

Não foram notificados acontecimentos adversos dos pares mãe/bebê no estudo de Emond et al. ${ }^{25}$ (2014). Das 99 frenotomias realizadas, quatro (4\%) precisaram ser repetidas porque o procedimento inicial não dividiu o frênulo suficientemente. Em 63 das frenotomias (64\%) havia uma pequena mancha branca na base do freio relatado pela mãe aos 5 dias, e estes levaram em média 7 dias (intervalo 1-30 dias) para cicatrizar.

É importante notar que, embora excepcionalmente rara, uma hemorragia grave com lesão dos vasos sublinguais ou edema submandibular pode ocorrer e, portanto, é sugerido que o procedimento deva ser realizado por profissionais devidamente treinados, dentro de um ambiente clínico onde um tratamento de urgência possa ser realizado ${ }^{32}$.

\section{Discussão}

Através da literatura consultada foi possível verificar que a prevalência de anquiloglossia entre bebês recém-nascidos é considerável, podendo chegar 
a $10 \%$ e que apesar dos diferentes pontos de vista apresentados por profissionais de diversas áreas sobre o diagnóstico, é necessário que este seja realizado nas maternidades, uma vez que pacientes portadores de tal anomalia poderão desenvolver problemas como alterações na amamentação, dicção, movimentação da língua e outras ${ }^{1,3,4}$.

Brito et al. ${ }^{3}$ (2008) realizaram estudo transversal com 90 profissionais (fonoaudiólogos, odontólogos e otorrinolaringologistas), com o objetivo de comparar a classificação e conduta em relação ao freio lingual. Quanto à classificação, foram observadas poucas divergências de opiniões. Entretanto, houve divergência de opinião quanto à conduta, pois alguns profissionais acreditam ser necessária a realização de fonoterapia após a cirurgia e outros acreditam que somente a cirurgia é capaz de corrigir os problemas causados pela anquiloglossia.

Dificuldades para diagnosticar a anquiloglossia foram relatadas em muitos estudos encontrados, pois não existe uma classificação padrão quanto aos tipos de alterações dos freios, prejudicando assim o consenso entre os profissionais em relação à conduta $^{33}$. Alguns autores, como Hazelbaker et al. ${ }^{7}$ (1993) e Marchesan $^{34}$ (2004), tentaram padronizar o diagnóstico através da elaboração de uma classificação. Amir et al. ${ }^{35}$ (2004) avaliaram a ferramenta proposta por Hazelbaker et al. ${ }^{7}$ (1993) e concluíram que a ferramenta possui alta aplicabilidade na prática clínica, porém seu uso para triagem poderia ser limitado por não ser de fácil aplicação.

O protocolo proposto por Martinelli et al. ${ }^{9}$ (2013) contém critérios para triagem e confirmação diagnóstica, porém apresenta algumas fragilidades, como a difícil aplicação; subjetividade da avaliação da amamentação, baseada no relato da mãe e em itens de observação não validados; os critérios de avaliação anatomo-funcional não foram validados e a prevalência de problemas apontada com a utilização do protocolo é superior ao identificado na literatura ${ }^{11}$.

O Bristol Tongue Assessment Tool (BTAT) ${ }^{10}$ propõe critérios para triagem e diagnóstico rápido de anquiloglossia grave. Trata-se de um protocolo validado e comparado ao de Hazelbaker ${ }^{7}$ (1993), e apresenta como vantagens a praticidade e objetividade na sua aplicação. Dos protocolos disponíveis, parece ser o mais adequado para realização da triagem neonatal ${ }^{11}$.

Grande parte dos estudos transversais e ensaios clínicos randomizados relatam que a frenotomia melhora a qualidade da amamentação dos bebês ${ }^{5,24-26,29}$, e que lactentes com anquiloglossia têm menor chance de serem amamentados nas primeiras semanas de vida, além de apresentarem maior risco de serem alimentados exclusivamente na mamadeira na primeira semana de vida comparados a lactentes sem anquiloglossia ${ }^{11}$.

Porém, as revisões sistemáticas acerca do tema $a^{2,28,33}$ não são precisas em sua conclusão, e ressaltam que a força de evidência é baixa, devido a pobre metodologia empregada nos estudos e as di- ficuldades inerentes à classificação do freio lingual, no entanto apontam para um efeito positivo da frenotomia sobre a melhora na amamentação.

Os efeitos adversos relatados em decorrência da frenotomia são mínimos, como sangramento leve ou formação de úlceras. No entanto, o procedimento deve ser feito por profissional de saúde treinado e em recém-nascidos com problemas significativos na amamentação em que houve falha do tratamento conservador $^{36}$.

$\mathrm{Na}$ literatura são encontrados diversos artigos que discorrem sobre a anquiloglossia e seu tratamento, entretanto em sua maioria são relatos de caso e estudos observacionais. Como já descrito anteriormente, não existe classificação padrão para a anquiloglossia, o que prejudica o consenso diagnóstico. Sugere-se a realização de mais estudos, sobretudo ensaios clínicos controlados, para que sejam construídas evidências científicas que embasem critérios diagnósticos e condutas.

\section{Considerações finais}

A anquiloglossia é uma anomalia que pode ser facilmente diagnosticada, mas que ainda gera controvérsias entre os profissionais quanto ao tratamento e quanto aos critérios de diagnóstico, ainda não bem definidos. Apesar das revisões sistemáticas não serem conclusivas, a maioria dos estudos apontam para uma melhora significativa na amamentação e relato de dor nos mamilos pelas mães após a realização da frenotomia, a qual não provoca complicações pós-cirúrgicas importantes. No entanto, as evidências ainda são fracas, pois mais estudos clínicos controlados precisam ser realizados, a fim de que estes achados possam ser mais conclusivos.

\section{Abstract}

Objective: this is study aimed to perform a literature review on the anomalous lingual frenulum in infants, relating diagnosis and treatment. Literature review: this anomaly limits tongue movements when crying and babbling, and may generate discomfort during breastfeeding. The methodology of the study consisted of searches of articles published in Portuguese and English in the Bireme, Google Scholar, and PubMed databases, between the years of 2004 and 2016. A total of 645 studies were identified through keyword combinations, and 29 of them were used because their results met the objective of this review. Final Considerations: the studies consulted did not allow identifying a gold-standard instrument to diagnose ankyloglossia in infants, despite the easy diagnose. There is controversy in the literature regarding the frenotomy treatment and its contribution to improving breastfeeding, tongue movements, and discomfort of the mother when breastfeeding, although a great portion of the studies shows obvious benefits.

Keywords: Lingual frenum. Ankyloglossia. Infants. 


\section{Referências}

1. Soares DRF, Ferrari LM, Faria MD. Anguiloglossia em bebês: relato de casos clínicos Rev Funec Cient Multidisc 2012;1:1-13.

2. Segal LM, Stephenson R, Dawes M, Feldman P. Prevalence, diagnosis, and treatment of ankyloglossia: methodologic review. Can Fam Physician 2007;53:1027-33.

3. Brito SF, Marchesan IQ, Bosco CM, Carrilho ACA, Rehder MI. Frênulo lingual: classificação e conduta segundo ótica fonoaudiológica, odontológica e otorrinolaringológica. Rev CEFAC 2008;10:343-51.

4. Melo NSFO, Lima AAS, Fernandes A, Silva RPGVC. Anquiloglossia: relato de caso. Rev Sul-Bras Odontol 2011;8:102-07.

5. Geddes DT, Langton DB, Gollow I, Jacobs LA, Hartmann PE, Simmer K. Frenulotomy for breastfeeding infants with ankyloglossia: effect on milk removal and sucking mechanism as imaged by ultrasound. Pediatrics 2008;122:e188-94.

6. Manfro AR, Manfro R, Bortoluzzi MC. Surgical treatment of ankyloglossia in babies-case report. Int J Oral Maxillofac Surg 2010;39(11):1130-2.

7. Hazelbaker AK. The assessment tool for lingual frenulum function (ATLFF): use in a lactation consultant private practice [Thesis]. Pasadena, California, MI: Pacific Oaks College; 1993.

8. Kotlow LA. Ankyloglossia (tongue-tie): a diagnostic and treatment quandary. Quintessence Int 1999;30:259-62.

9. Martinelli RLCM, Marchesan IQ; Berretin-Felix G. Protocolo de avaliação do frênulo lingual para bebês: relação entre aspectos anatômicos e funcionais. Rev CEFAC 2013;15:599-610.

10. Ingram J, Johnson D, Copeland M, Churchill C, Taylor H, Emond A. The development of a tongue assessment tool to assist with tongue-tie identification. Arch Dis Child Fetal Neonatal Ed 2015;100:F344-8.

11. Venancio SI, Toma TS, Buccini GS, Sanches MTC, Araújo CL, Figueiró MF. Anquiloglossia e aleitamento materno: evidências sobre a magnitude do problema, protocolos de avaliação, segurança e eficácia da frenotomia. Parecer Técnico-Científico- Instituto de Saúde. São Paulo; 2015.

12. Ten Cate NA. Histologia oral: desenvolvimento, estrutura e função. Rio de Janeiro: Elsevier Health Sciences; 2008.

13. Morita H, Mazerbourg S, Bouley DM, Luo CW, Kawamura K, Kuwabara Y, et al. Neonatal lethality of LGR5 null mice is associated with ankyloglossia and gastrointestinal distension. Mol Cell Biol 2004;24:9736-43.

14. WHO. Guiding principles for complementary feeding of the breastfeed child. [cited 2016 Dec 18]. Available from: www. who.int/.../guiding_principles_compfeeding_breastfed.pdf.

15. Sanches MTC. Manejo clínico das disfunções orais na amamentação. J Pediatr 2004;80:155-62.

16. Isaacson GC, Messner AH, Arsmby C. Ankyloglossia (tonguetie) in infants and children. [cited 2016 Dec 02]. Available from: http://www.uptodate.com.

17. Silva MC, da Costa MLVCM, Nemr K, Marchesan IQ. Frênulo de língua alterado e interferência na mastigação. Rev CEFAC 2009;11:363-69.

18. Salem G, Holm SA, Fattah R, Basset S, Nasser C. Developmental oral anomalies among schoolchildren in Gizan region, Saudi Arabia. Community Dent Oral Epidemiol 1987;15:150-1.

19. Hogan M, Westcott C, Griffiths M. Randomized, controlled trial of division of tongue-tie in infants with feeding problems. J Paediatr Child Health 2005;41:246-50.
20. Messner AH, Lalakea ML, Aby J, Macmahon J, Bair E. Ankyloglossia: incidence and associated feeding difficulties. Arch Otolaryngol Head Neck Surg 2000;126:36-9.

21. Ricke LA, Baker NJ, Madlon-Kay DJ, DeFor TA. Newborn tongue-tie: prevalence and effect on breast-feeding. J Am Board Fam Pract 2005;18:1-7.

22. Peterson LJ. Cirurgia Oral e Maxilofacial Contemporânea. 5 ed. Rio de Janeiro: Elsevier Brasil; 2009.

23. Marchesan IQM, Martinelli RLC, Gusmão RJ. Frênulo lingual: modificações após frenectomia. J Soc Bras Fonoaudiol 2012;24:409-12.

24. Berry J, Griffiths M, Westcott C. A double-blind, randomized, controlled trial of tongue-tie division and its immediate effect on breastfeeding. Breastfeed Med 2012;7:189-93.

25. Emond A, Ingram J, Johnson D, Blair P, Whitelaw A, Copeland M, et al. Randomised controlled trial of early frenotomy in breastfed infants with mild-moderate tongue-tie. Arch Dis Child Fetal Neonatal Ed 2014;99:F189-95.

26. Haham A, Marom R, Mangel L, Botzer E, Dollberg S. Prevalence of breastfeeding difficulties in newborns with a lingual frenulum: a prospective cohort series. Breastfeed Med 2014;9(9):438-41.

27. Coryllos EGC, Salloum AC. American Academy of Pediatrics Section on Breastfeeding. [cited 2016 Nov 24]. Available from: www2.aap.org/breastfeeding/files/pdf/BBM-8-27\%20 Newsletter.pdf.

28. Francis DO, Krishnaswami S, McPheeters M. Treatment of ankyloglossia and breastfeeding outcomes: a systematic review. Pediatrics 2015;135:e1458-66.

29. Yousefi J, Tabrizian Namini F, Raisolsadat SM, Gillies R, Ashkezari A, Meara JG. Tongue-tie Repair: Z-Plasty Vs Simple Release. Iran J Otorhinolaryngol 2015;27:127-35.

30. Braga LAS, da Silva J, Pantuzzo CL, Motta AR. Prevalência de alteração no frênulo lingual e suas implicações na fala de escolares. Rev CEFAC 2009;11:378-90.

31. Griffiths DM. Do tongue ties affect breastfeeding? J Hum Lact 2004;20:409-14.

32. Opara PI, Gabriel-Job N, Opara KO. Neonates presenting with severe complications of frenotomy: a case series. J Med Case Rep 2012;6:77.

33. Suter VG, Bornstein MM. Ankyloglossia: facts and myths in diagnosis and treatment. J Periodontol 2009;80:1204-19.

34. Marchesan IQ. Frênulo lingual: proposta de avaliação quantitativa. Rev CEFAC 2004;6:288-93.

35. Amir LH, James JP, Donath SM. Reliability of the hazelbaker assessment tool for lingual frenulum function. Int Breastfeed J 2006; $1: 3$.

36. Webb AN, Hao W, Hong P. The effect of tongue-tie division on breastfeeding and speech articulation: a systematic review. Int J Pediatr Otorhinolaryngol 2013;77:635-46.

Endereço para correspondência:

Vanessa Polina Pereira Costa

UnB - Campus Darcy Ribeiro

Faculdade de Ciências da Saúde - Departamento de Odontologia

Cep: 70910-900 - Asa Norte - Brasília - DF

Fone: (61) 998635968

email: polinatur@yahoo.com.br

Recebido: 12/01/2017. Aceito: 22/03/2017. 